\title{
Complicated Laparoscopic Cholecystectomy
}

\author{
A Case Report \\ D.J . Gouma
}

Department of Surgery, Academic Medical Center at the University of Amsterdam, The Netherlands

\section{Key Words}

Bile duct injury · Bile leakage - Cholecystectomy . Laparoscopic surgery $\cdot$ Biloma B Biliary strictures

\section{Case Report}

A 42-year-old male was admitted in a community hospital because of symptomatic gallstone disease (recurrent attacks of abdominal pain RUQ), without jaundice. Ultrasound confirmed gallstones and the common bile duct (CBD) was not dilated. A laparoscopic cholecystectomy was performed and during the procedure, after clipping the cystic artery, a severe bleeding occurred. The procedure was converted to an open procedure. The bleeding site was ligated successfully. The surgeon mentioned in the operative report that the bleeding site might have been the hepatic artery. The recovery was uneventful and the patient was discharged 2 days postoperatively.

Three months later he was readmitted with abdominal pain and obstructive jaundice confirmed by liver function tests (alkaline phosphatase $352 \mathrm{U} / 1, \gamma$-GT $400 \mathrm{U} / \mathrm{l}$, SGOT $122 \mathrm{U} / \mathrm{l}$, SGPT $272 \mathrm{U} / 1$, serum bilirubin $48 \mu \mathrm{mol} / \mathrm{l})$.

\section{Question}

Which diagnosis is most likely?
A Retained CBD stone
B CBD stenosis/injury
C Liver ischemia (ligation hepatic artery)
D Proximal bile duct tumor (initially missed)

\section{Comment}

The most likely diagnosis is a CBD stenosis probably due to an ischemic injury of the arterial supply of the bile duct considering the time interval between the procedure and development of the symptoms. A retained stone is a second choice, however unlikely, because the patient did not have a history of jaundice or liver function abnormalities before surgery, and the preoperative ultrasound showed no dilatation of the bile ducts. Liver ischemia or isolated ischemia of the right lobe 3 months after ligation of the hepatic artery without previous complaints is unlikely because of the time interval.

Case Report (Continued)

After readmission, ultrasound showed dilatation of the intrahepatic bile ducts and a fluid collection outside the liver was found.

\section{Question}

Which diagnostic or therapeutic procedure do you prefer?
A Laparotomy/exploration
B Puncture fluid collection
$28 \%$
C MRCP
$32 \%$
D ERCP/PTC
$10 \%$
$30 \%$

Audience's

\begin{tabular}{ll}
\hline KARGER & ๑ 2002 S. Karger AG, Basel \\
Fax +41 61 306 12 34 & 0253-4886/02/0192-0114\$18.50/0 \\
$\begin{array}{l}\text { E-Mail karger@karger.ch } \\
\text { www.karger.com }\end{array}$ & $\begin{array}{l}\text { Accessible online at: } \\
\text { www.karger.com/journals/dsu }\end{array}$
\end{tabular}

\author{
D. J. Gouma \\ Department of Surgery, Academic Medical Center \\ P.O. Box 22700 \\ NL-1100 DD Amsterdam (The Netherlands) \\ Tel. +3120 5662166, Fax +3120 5669243, E-Mail d.j.gouma@amc.uva.nl
}


Fig. 1. ERCP 3 months after laparoscopic cholecystectomy showing stenosis of the proximal bile duct, bile leakage and a fluid collection. The intrahepatic ducts (right side) are not visualized in detail.

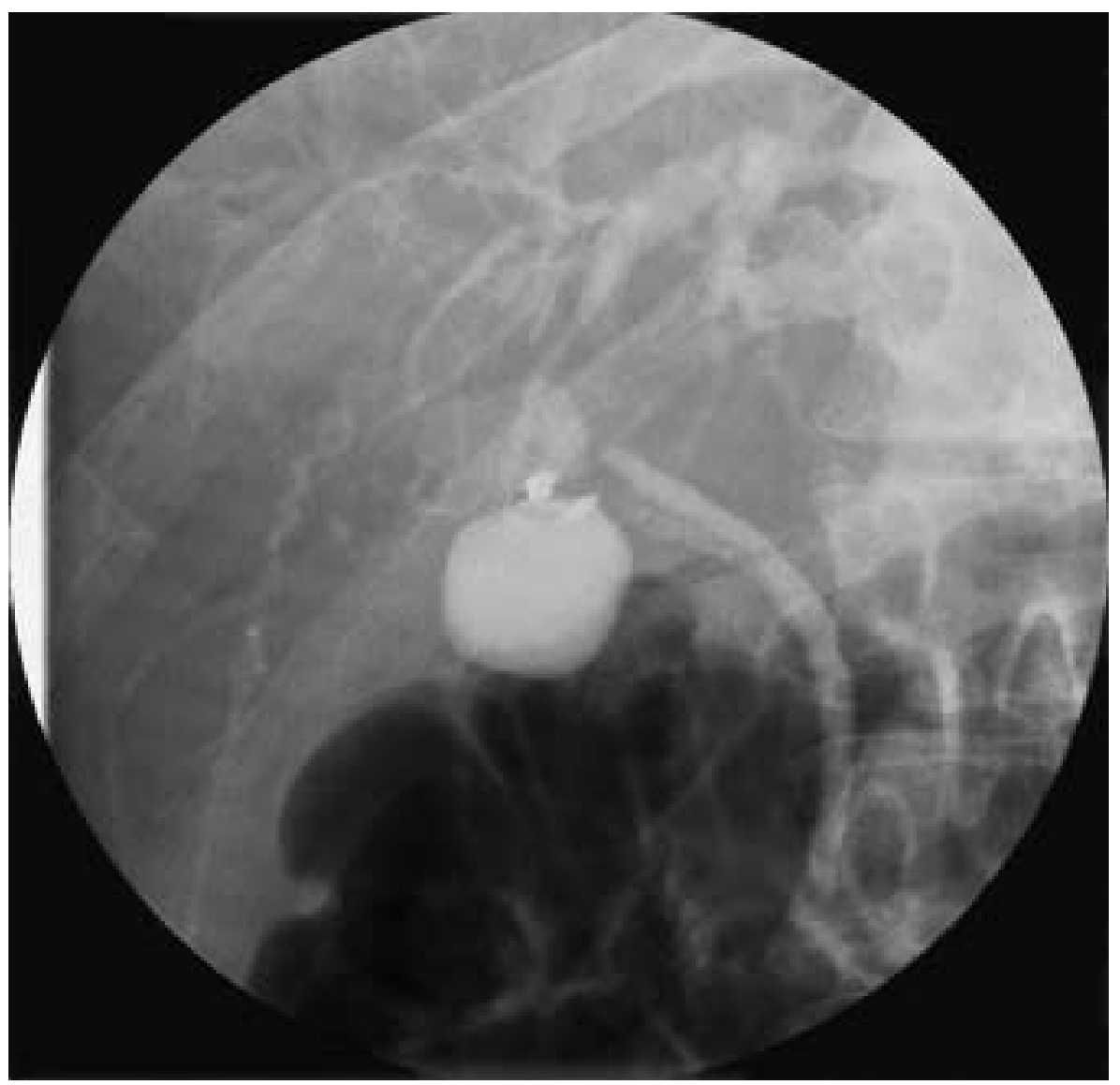

\section{Comment}

Most importantly, a laparotomy (still 28\%) should be avoided because at this point there is no classification of the severity of the injury; the anatomy of the biliary tract is not visualized and there is not a well-defined plan for repair. One could consider MRCP as the next, noninvasive test, if available, but the other procedures are both well accepted.

\section{Case Report (Continued)}

A puncture of the fluid collection was not possible because of the size and location $(2 \times 3 \mathrm{~cm})$, MRCP was not available and therefore ERCP was performed and a stenosis of the bile duct, bile leakage and a fluid collection was shown with an inadequate filling of the intrahepatic ducts in particular the right duct (fig. 1). Subsequently a PTC was performed showing an irregular stenosis at the confluence with bile leakage. External drainage was performed (fig. 2a, b). Two days later there was dislodgment of the drain and progressive abdominal pain. The patient was referred to our institution.

\section{Summary after Referral to the AMC}

A 42-year-old male with progressive abdominal pain, fever $38.2^{\circ} \mathrm{C}$, mild jaundice and dislodgment of the PTC catheter in a patient with a stenosis/defect of the bile duct at the confluence and bile leakage 3 months after laparoscopic cholecystectomy.

\section{Question}

What is your next diagnostic/therapeutic step?

\section{A Exploration/reconstruction}

Audience's response

B Angiography (hepatic artery) $60 \%$

C PTC and internal drainage/dilatation $10 \%$

D PTC and delayed reconstruction $5 \%$ $25 \%$

\section{Comment}

The arguments to perform exploration are that the extent of lesion is clearly shown by ERCP/PTC and during previous PTC and ERCP it was not possible to pass 

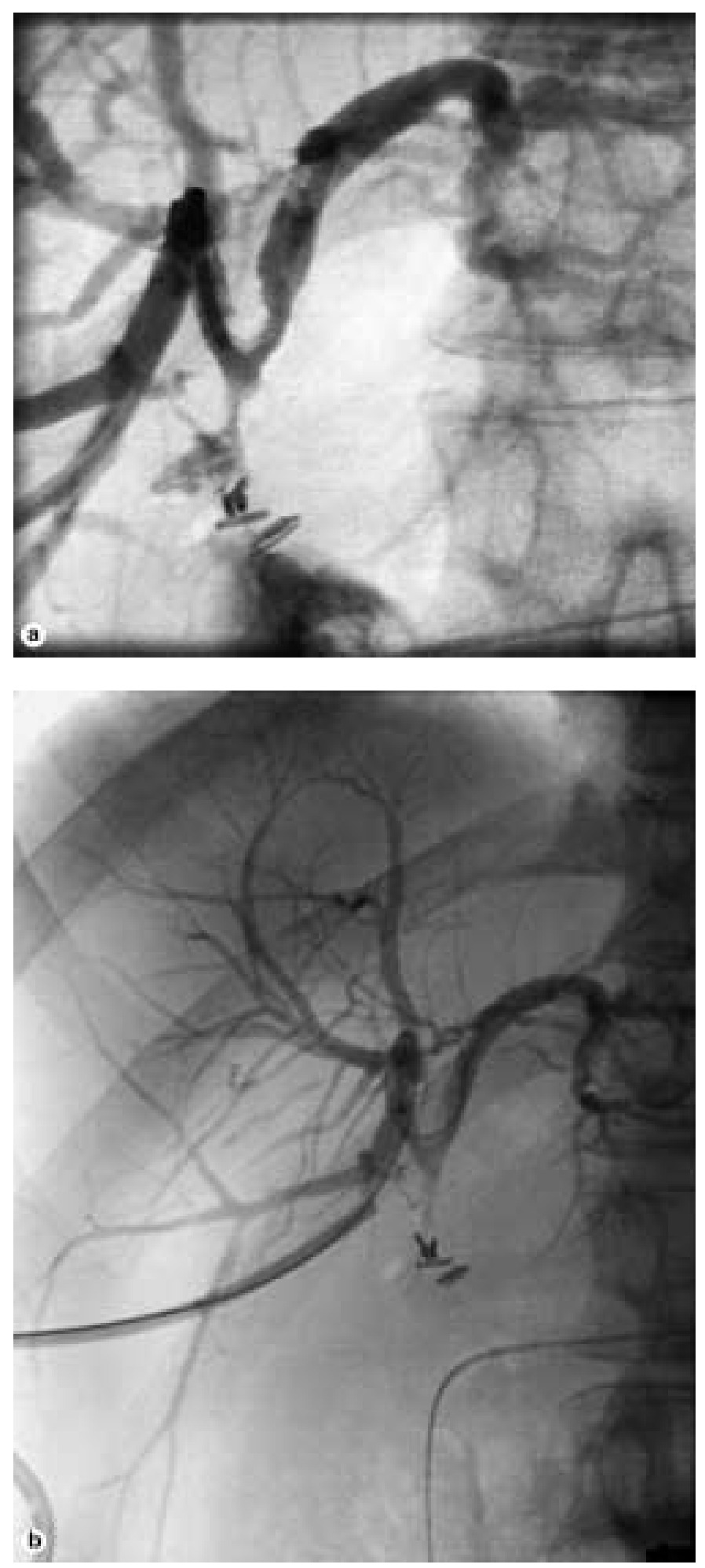

Fig. 2. PTC 3 months after laparoscopic cholecystectomy showing an irregular stenosis at the confluence with bile leakage. a Detail of hilus. b Overview.

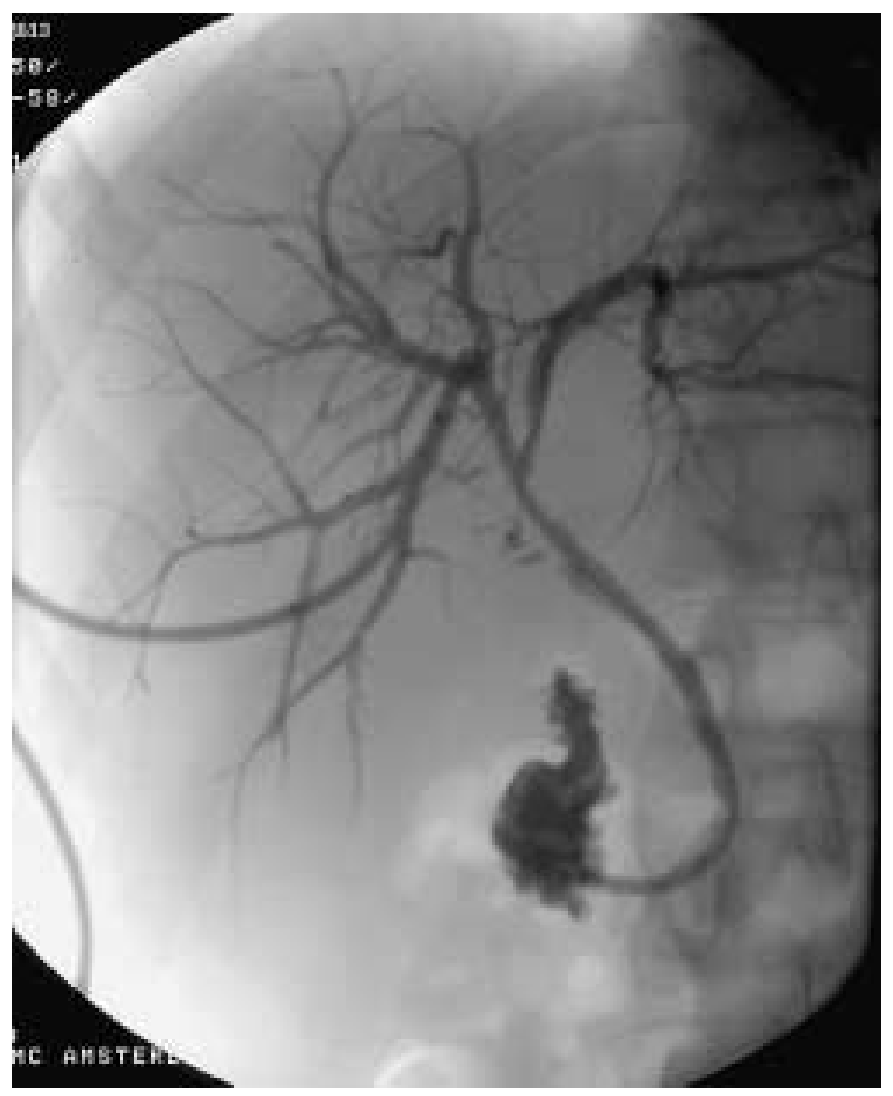

Fig. 3. PTC 3 months after laparoscopic cholecystectomy. The defect stenosis in the proximal bile duct is passed and internal biliary drainage is performed.

the stricture. The chance for successful internal drainage is therefore limited. Findings on a subsequent angiography will not change the therapeutic strategy despite the fact that abnormalities will be found in $25 \%$.

The arguments in favor of a second PTC are that the patient is suffering from active biliary leakage, with a fluid collection and that results of an elective reconstruction later without the presence of leakage/inflammation are better compared with a subacute reconstruction.

\section{Case Report (Continued)}

A rendezvous PTC/ERCP was performed with subsequent internal drainage (fig. 3). Due to the extent of the lesion in the bile duct, long-term drainage and pneumodilatation were considered not to be highly successful. An elective reconstruction by hepaticojejunostomy at the confluence was performed 6 weeks later. The patient was discharged after 5 days and has currently no complaints and normal liver function tests. 